\title{
MAPPINGS OF FINITE DISTORTION: GENERALIZED HAUSDORFF DIMENSION DISTORTION
}

\author{
PEKKA KOSKELA, ALEKSANDRA ZAPADINSKAYA, \\ AND THOMAS ZÜRCHER
}

\begin{abstract}
We study how planar Sobolev homeomorphisms distort sets of Hausdorff dimension strictly less than two. We measure the image size by means of a generalized Hausdorff measure. As an application, we obtain a sharp generalized dimension distortion estimate for mappings of exponentially integrable distortion.
\end{abstract}

\section{INTRODUCTION}

Let $f: \Omega \rightarrow \mathbb{R}^{2}$ be a continuous mapping, where $\Omega \subset \mathbb{R}^{2}$. We say that $f$ has finite distortion if $f \in W_{\mathrm{loc}}^{1,1}\left(\Omega ; \mathbb{R}^{2}\right), J_{f} \in L_{\mathrm{loc}}^{1}(\Omega)$ and there exists a measurable function $K$ such that $1 \leq K(x)<\infty$ a.e. in $\Omega$ and $|D f(x)|^{2} \leq K(x) J_{f}(x)$ a.e. in $\Omega$. When $K$ is bounded, we obtain the class of mappings of bounded distortion, also called quasiregular mappings. In this case, the image of any set of Hausdorff dimension strictly less than two under $f$ is of the same class, and sets of area zero are mapped to sets of area zero. This result relies on the higher integrability results for the Jacobian of a quasiregular mapping, see [Boy57], [GV73].

We will concentrate on the case when $\exp (\lambda K) \in L_{\text {loc }}^{1}(\Omega)$ for some $\lambda>0$. For short, we declare that $f$ is of locally $\lambda$-exponentially integrable distortion. For the basic properties of these mappings we refer the reader to [IKO01, KKM01b, KKM01a] and for the existence theory to [Dav88, IM01, IM08]. Similarly as for mappings of bounded distortion, sets of area zero are mapped to sets of area zero. However, a set, for example, of dimension one can be mapped onto a set of Hausdorff dimension two. Our main result gives a rather sharp estimate on the size of the image set.

Theorem 1. Let $f \in W_{\text {loc }}^{1,1}\left(\Omega ; \mathbb{R}^{2}\right), \Omega \subset \mathbb{R}^{2}$, be a mapping of locally $\lambda$-exponentially integrable distortion, $\lambda>0$. Set $h_{s}(t)=t^{2} \log ^{s}(1 / t)$ for $s \in \mathbb{R}$. If $E \subset \mathbb{R}^{2}$ satisfies $\operatorname{dim}_{\mathcal{H}}(E)<2$, then $\mathcal{H}^{h_{s}}(f(E))=0$ for all $s<\lambda$, where $\mathcal{H}^{h_{s}}$ is the generalized Hausdorff measure associated to $h_{s}$.

2000 Mathematics Subject Classification. Primary 30C65.

The first two authors were supported by the Academy of Finland, grant no. 120972, and the third author was supported by the Swiss National Science Foundation and GALA. 
Theorem 1 is essentially sharp. Indeed, by Proposition 5.1 in [HK03], for any given $\varepsilon>0$ and $\lambda>0$ we can find $f$, having locally $(\lambda-\varepsilon)$ exponentially integrable distortion, and mapping a set of Hausdorff dimension strictly less than two onto a set of positive generalized Hausdorff measure with the gauge function $h(t)=t^{2} \log ^{\lambda}(1 / t)$. Partial motivation for Theorem 1 comes from trying to estimate the size of the image of the unit circle under a mapping of locally exponentially integrable distortion. In the case of bounded distortion these images are called quasicircles and the question is rather well understood. Theorem 1 improves on the previous estimate from [HK03].

In the case of a mapping of bounded distortion, the dimension distortion estimates follow from the higher regularity of the mappings in question. Indeed, if $f$ belongs to the Sobolev space $W^{1, p}\left(\Omega ; \mathbb{R}^{2}\right), p>2$, then $f$ maps sets of Hausdorff-dimension strictly less than two to sets of the same type [GV73], [Kau00]. This may fail when $f \in W^{1,2}\left(\Omega ; \mathbb{R}^{2}\right)$ : one can even map a Cantor set of dimension, for example, one onto a set of positive area. However, if $f \in W^{1,2}\left(\Omega ; \mathbb{R}^{2}\right)$ is injective, then $f$ maps sets of area zero to sets of area zero by results of Reshetnyak [Reš66]. Our next result shows that a logarithmic improvement on the $L^{2}$-integrability of the differential results in generalized dimension bounds.

Theorem 2. Let $\Omega$ be an open set in $\mathbb{R}^{2}$ and $f: \Omega \rightarrow f(\Omega) \subset \mathbb{R}^{2}$ be a homeomorphism in $W^{1,2}\left(\Omega ; \mathbb{R}^{2}\right)$ with $|D f|^{2} \log ^{\lambda}(e+|D f|) \in L_{\text {loc }}^{1}(\Omega)$ for some $\lambda>0$. Then, for $E \subset \mathbb{R}^{2}$, we have

$$
\operatorname{dim}_{\mathcal{H}}(E)<2 \Longrightarrow \mathcal{H}^{h}(f(E))=0
$$

for $h(t)=t^{2} \log ^{\lambda}\left(\frac{1}{t}\right)$.

We do not know if the estimate in Theorem 2 is sharp, see however [KZZ] for a related result for Minkowski dimension. The proof of Theorem 1 is based on the potentially non-sharp estimate from Theorem 2, the higher regularity of our mappings and a suitable factorization argument. Indeed, a mapping $f$ of locally exponentially integrable distortion is a priori only in the class $W_{\text {loc }}^{1,1}\left(\Omega ; \mathbb{R}^{2}\right)$ but in fact $|D f|^{2} \log ^{c \lambda-1}(e+|D f|) \in L_{\text {loc }}^{1}(\Omega)$ with a universal $c$ [Dav88, IKM02, IKMS03, FKZ05]. Recently, it has been proved that this holds for all $c<1$ [AGRS, Theorem 1.1]. This sharp estimate together with a usual factorization of our mapping $f$ from Theorem 1 into a homeomorphism and a holomorphic function together with Theorem 2 rather easily gives a weaker version of Theorem 2, with a worse exponent of the logarithm in the definition of $h$ than indicated. We establish the sharp bound by employing a slightly more complicated decomposition.

The paper is organized as follows. Section 2 contains some preliminaries. Theorem 2 is proven in Section 3. Finally, Section 4 contains the proof of Theorem 1 . 
Acknowledgments. A large part of this research took place while the third author was visiting the University of Jyväskylä. He would like to thank the University and especially the department of mathematics and statistics for its hospitality and its support.

\section{Preliminaries}

We write $\mathcal{H}^{h}(A)$ for the generalized Hausdorff measure of a set $A$, given by

$$
\begin{aligned}
\mathcal{H}^{h}(A)= & \lim _{\delta \rightarrow 0} \mathcal{H}_{\delta}^{h}(A) \\
& =\lim _{\delta \rightarrow 0}\left[\inf \left\{\sum_{i=1}^{\infty} h\left(\operatorname{diam} U_{i}\right): A \subset \bigcup_{i=1}^{\infty} U_{i}, \operatorname{diam} U_{i} \leq \delta\right\}\right],
\end{aligned}
$$

where $h$ is a dimension gauge (non-decreasing, $h(0)=0$ ). If $h(t)=t^{\alpha}$ for some $\alpha \geq 0$, we put simply $\mathcal{H}^{\alpha}$ for $\mathcal{H}^{t^{\alpha}}$ and call it the Hausdorff $\alpha$-dimensional measure and the Hausdorff dimension $\operatorname{dim}_{\mathcal{H}}(A)$ of the set $A$ is the smallest $\alpha_{0} \geq 0$ such that $\mathcal{H}^{\alpha}(A)=0$ for any $\alpha>\alpha_{0}$.

We will compare integrals over annuli with integrals over circles. For $x \in \mathbb{R}^{2}$ and $0<r<R$, we will use the symbol $A(x, r, R)$ to denote the closed annulus with center at $x$ and radii $r$ and $R$ :

$$
A(x, r, R)=\left\{y \in \mathbb{R}^{2}: r \leq|x-y| \leq R\right\} .
$$

We will denote by $S^{1}(x, r)$ the circle with center at $x$ and radius $r$.

Finally, we will need the following concept of a maximal operator. Assume that $\Omega$ is a square and $h: \Omega \rightarrow \mathbb{R}$ is nonnegative and integrable. The maximal operator $\mathcal{M}_{\Omega}$ is defined by

$$
\mathcal{M}_{\Omega} h(x)=\sup \left\{f_{Q} h d x: x \in Q \subset \Omega\right\}
$$

where the supremum is taken over all subsquares of $\Omega$ containing the given point $x \in \Omega$.

\section{Proof of Theorem 2}

We begin by giving a short strategy of the proof of Theorem 2. First, we show that

$$
\operatorname{diam} f(B(x, \vartheta r)) \leq \frac{C(\vartheta)}{r} \int_{B(x, r)} \mathcal{M}_{\Omega}|D f|(y) d y
$$

for $\vartheta>1 / 2$, where $C(\vartheta)$ is a constant depending only on $\vartheta$, and $\mathcal{M}_{\Omega}$ denotes the maximal function defined above. The dependence on $\vartheta$ is also some sort of hidden in the maximal function. Note that the two balls in the inequality have different sizes. The reader who is familiar with the $5 r$-Covering Theorem may notice that this is a nice situation 
for this theorem to kick in. We break the proof of (2) into two major parts. The first step is to establish the inequality

$$
\operatorname{diam} f(B(x, \vartheta r)) \leq \frac{1}{r} \int_{A(x, \vartheta r, 3 \vartheta r)}|D f(y)| d y
$$

by averaging the inequality

$$
\operatorname{diam} f(B(x, \vartheta r)) \leq \int_{S^{1}(x, t)}|D f(y)| d s_{y}
$$

which holds for almost every $t \in[\vartheta r, 3 \vartheta r]$. The second step is to prove that

$$
\frac{1}{r} \int_{A(x, \vartheta r, 3 \vartheta r)}|D f(u)| d u \leq \frac{C}{r} \int_{B(x, r)} \mathcal{M}_{\Omega}(|D f|)(y) d y .
$$

Having diam $f(B(x, \vartheta r))$ under control, we control

$$
\operatorname{diam}^{2} f(B(x, \vartheta r)) \log ^{\lambda}\left(\frac{1}{\operatorname{diam} f(B(x, \vartheta r))}\right)
$$

by terms of the form

$$
\log ^{\lambda}\left(\frac{1}{\int_{B(x, r)} \mathcal{M}_{\Omega}^{2}(|D f|)(y) d y}\right) \int_{B(x, r)} \mathcal{M}_{\Omega}^{2}(|D f|)(y) d y .
$$

To end the proof, we choose a nice covering of $E$ and find upper bounds for the terms in (6) by classifying the balls resulting into different groups.

We conclude (3) by the following lemma.

Lemma 1. Let $\Omega \subset \mathbb{R}^{2}$ be an open square and $f: \Omega \rightarrow f(\Omega)$ be a homeomorphism in $W^{1,1}\left(\Omega, \mathbb{R}^{2}\right), x \in \Omega, r>0$ and $\vartheta>1 / 2$ such that that $B(x, 3 \vartheta r) \subset \Omega$. Then

$$
\operatorname{diam} f(B(x, \vartheta r)) \leq \frac{1}{r} \int_{A(x, \vartheta r, 3 \vartheta r)}|D f(y)| d y .
$$

Proof. As the mapping $f$ is a homeomorphism, we have

$$
\operatorname{diam} f(B(x, \vartheta r)) \leq \operatorname{diam} f(\partial B(x, t))=\operatorname{diam} f\left(S^{1}(x, t)\right)
$$

for all $t \in[\vartheta r, 3 \vartheta r]$. So, in order to prove (4), it suffices to establish

$$
\operatorname{diam} f\left(S^{1}(x, t)\right) \leq \int_{S^{1}(x, t)}|D f(y)| d s_{y}
$$

for $\mathcal{L}^{1}$-almost every $t \in[\vartheta r, 3 \vartheta r]$. Clearly, this estimate is true for smooth mappings. Let us take componentwise the standard smooth approximations $f^{\varepsilon}$ of $f$ in $\Omega$. As the limit mapping $f$ is continuous, the convergence $f^{\varepsilon} \rightarrow f$, when $\varepsilon \rightarrow 0$, is pointwise and uniform on each compact set $K \subset \Omega$. Thus, for $t \in[\vartheta r, 3 \vartheta r$, we have

$$
\operatorname{diam} f\left(S^{1}(x, t)\right)=\lim _{\varepsilon \rightarrow 0} \operatorname{diam} f^{\varepsilon}\left(S^{1}(x, t)\right) \leq \liminf _{\varepsilon \rightarrow 0} \int_{S^{1}(x, t)}\left|D f^{\epsilon}(y)\right| d s_{y} .
$$


On the other hand, we have the convergence $D f^{\epsilon} \rightarrow D f$, when $\varepsilon \rightarrow 0$, in $L^{1}(\Omega)$. That is, integration in polar coordinates gives us

$$
\int_{[\vartheta r, 3 \vartheta r]} \int_{S^{1}(x, t)}\left|D f^{\epsilon}(y)-D f(y)\right| d s_{y} d t \rightarrow 0,
$$

when $\varepsilon \rightarrow 0$. Passing to a subsequence $\left\{f_{j}\right\}_{j=1}^{\infty} \subset\left\{f^{\varepsilon}: \varepsilon>0\right\}$ lets us conclude

$$
\int_{S^{1}(x, t)}\left|D f^{j}(y)-D f(y)\right| d s_{y} \rightarrow 0
$$

when $j \rightarrow \infty$, for almost every $t \in[\vartheta r, 3 \vartheta r]$. Next, we obtain

$$
\begin{aligned}
\limsup _{j \rightarrow \infty}\left|\int_{S^{1}(x, t)}\right| D f^{j}(y)\left|d s_{y}-\int_{S^{1}(x, t)}\right| D f(y)\left|d s_{y}\right| \\
\leq \limsup _{j \rightarrow \infty} \int_{S^{1}(x, t)}\left|D f^{j}(y)\right|-|D f(y)| \mid d s_{y} \\
\leq \limsup _{j \rightarrow \infty} \int_{S^{1}(x, t)}\left|D f^{j}(y)-D f(y)\right| d s_{y}=0
\end{aligned}
$$

for almost every $t \in[\vartheta r, 3 \vartheta r]$. This together with (7) and (8) gives us

$$
\operatorname{diam} f(B(x, \vartheta r)) \leq \int_{S^{1}(x, t)}|D f(y)| d s_{y}
$$

for $\mathcal{L}^{1}$-almost every $t \in[\vartheta r, 3 \vartheta r]$. Finally, integrating this estimate over $[\vartheta r, 3 \vartheta r]$ with respect to $t$, we arrive at

$$
\begin{aligned}
2 \vartheta r \operatorname{diam} f(B(x, \vartheta r)) & \leq \int_{[\vartheta r, 3 \vartheta r]} \int_{S^{1}(x, t)}|D f(y)| d s_{y} d t \\
& =\int_{A(x, \vartheta r, 3 \vartheta r)}|D f(y)| d y .
\end{aligned}
$$

Taking into consideration the fact that $\vartheta>1 / 2$ finishes the proof.

Now we tackle (5).

Lemma 2. Let $\Omega \subset \mathbb{R}^{2}$ be a square, $f: \Omega \rightarrow \mathbb{R}, f \in W^{1,2}\left(\Omega ; \mathbb{R}^{2}\right)$, and $\vartheta>1 / 2$. Finally assume that $B(x,(2+3 \vartheta) \sqrt{2} r)$ is contained in $\Omega$. Then there is a constant $C=C(\vartheta)>0$ such that

$$
\int_{A(x, \vartheta r, 3 \vartheta r)}|D f(y)| d y \leq C \int_{B(x, r)} \mathcal{M}_{\Omega}|D f|(y) d y .
$$

Proof. For $y \in \mathbb{R}^{2}$ and $\rho>0$, let $Q(y, \rho)$ denote the square

$$
Q(y, \rho):=\left\{z \in \mathbb{R}^{2}: \max _{i \in\{1,2\}}\left(\left|z_{i}-y_{i}\right|\right) \leq \rho\right\} .
$$

The key of the proof lies in the transition from the integral over $Q(y,(1+3 \vartheta) r), y \in B(x, r)$, to the one over $A(x, \vartheta r, 3 \vartheta r)$. The square 
is chosen in such a way that it is contained in $\Omega$ and contains the annulus. Now

$$
\begin{aligned}
\int_{B(x, r)} \mathcal{M}_{\Omega} \mid & D f \mid(y) d y \\
& \geq \frac{1}{((1+3 \vartheta) r)^{2}} \int_{B(x, r)} \int_{Q(y,(1+3 \vartheta) r)}|D f(z)| d z d y \\
& \geq \frac{1}{((1+3 \vartheta) r)^{2}} \int_{B(x, r)} \int_{A(x, \vartheta r, 3 \vartheta r)}|D f(z)| d z d y \\
& =\frac{\mathcal{L}^{2}(B(x, r))}{((1+3 \vartheta) r)^{2}} \int_{A(x, \vartheta r, 3 \vartheta r)}|D f(z)| d z \\
& =C(\vartheta) \int_{A(x, \vartheta r, 3 \vartheta r)}|D f(z)| d z
\end{aligned}
$$

The next lemma is basically a special case of Lemma 5.1 in [GIM95].

Lemma 3. Let $\Omega \subset \mathbb{R}^{2}$ be an open square and $f: \Omega \rightarrow \mathbb{R}$ be in $W^{1,2}\left(\Omega ; \mathbb{R}^{2}\right)$ with the property $|D f|^{2} \log ^{\lambda}(e+|D f|) \in L^{1}(\Omega)$ for some $\lambda>0$. Then

$$
\mathcal{M}_{\Omega}^{2}(|D f|) \log ^{\lambda}\left(e+\mathcal{M}_{\Omega}^{2}(|D f|)\right) \in L^{1}(\Omega) .
$$

Proof. We apply Lemma 5.1 from [GIM95] for $n=2, h=|D f|$ and

$$
\Phi(t)=A(t) t^{2}=t^{2} \log ^{\lambda}(t+e),
$$

obtaining

$$
\begin{gathered}
\int_{\Omega} \mathcal{M}_{\Omega}^{2}(|D f|)(y) \log ^{\lambda}\left(e+\mathcal{M}_{\Omega}^{2}(|D f|)(y)\right) d y \\
\leq \int_{\Omega}(C|D f(y)|)^{2} \log ^{\lambda}(e+C|D f(y)|) d y \\
\leq \tilde{C} \int_{\Omega}|D f(y)|^{2} \log ^{\lambda}(e+|D f(y)|) d y
\end{gathered}
$$

where $C>0$ and $\tilde{C}=\tilde{C}(\lambda)>0$ are constants.

Before we turn to bounds for images of sets of small Hausdorff measure, we state a result that controls the images of balls.

Proposition 1. Let $\Omega \subset \mathbb{R}^{2}$ be a square and $f: \Omega \rightarrow f(\Omega)$ be a homeomorphism in $W^{1,2}\left(\Omega ; \mathbb{R}^{2}\right)$ with the property

$$
|D f|^{2} \log ^{\lambda}(e+|D f|) \in L^{1}(\Omega)
$$


for some $\lambda>0$. Fix $\vartheta>1 / 2$. Then there is a constant $L=L(\vartheta, \lambda)$ and for every $x \in \Omega$ a positive $R_{x}$ such that

$$
\begin{aligned}
& (\operatorname{diam} f(B(x, \vartheta r)))^{2} \log ^{\lambda}\left(\frac{1}{\operatorname{diam} f(B(x, \vartheta r))}\right) \\
& \quad \leq L \log ^{\lambda}\left(\frac{1}{\int_{B(x, r)} \mathcal{M}_{\Omega}^{2}(|D f|)(z) d z}\right) \int_{B(x, r)} \mathcal{M}_{\Omega}^{2}(|D f|)(y) d y
\end{aligned}
$$

for all $0<r<R_{x}$.

Proof. Fix $x \in \Omega$ and $r>0$ such that $B(x,(2+3 \vartheta) \sqrt{2} r) \subset \Omega$. In Lemma 1, we checked that

$$
\operatorname{diam} f(B(x, \vartheta r)) \leq \frac{1}{r} \int_{A(x, \vartheta r, 3 \vartheta r)}|D f(y)| d y .
$$

By combining this inequality with Lemma 2, we obtain the estimate

$$
\operatorname{diam} f(B(x, \vartheta r)) \leq \frac{C(\vartheta)}{r} \int_{B(x, r)} \mathcal{M}_{\Omega}(|D f|)(y) d y .
$$

In the following, $C \geq 1$ is a constant whose value may vary from formula to formula, but it only depends on $\vartheta$ and $\lambda$. By applying the Cauchy-Schwarz inequality, we obtain

$$
\begin{aligned}
(\operatorname{diam} f(B(x, \vartheta r)))^{2} & \leq \frac{C}{r^{2}}\left(\int_{B(x, r)} \mathcal{M}_{\Omega}(|D f|)(y) d y\right)^{2} \\
& \leq \frac{C}{r^{2}} \mathcal{L}^{2}(B(x, r)) \int_{B(x, r)} \mathcal{M}_{\Omega}^{2}(|D f|)(y) d y \\
& \leq C \int_{B(x, r)} \mathcal{M}_{\Omega}^{2}(|D f|)(y) d y .
\end{aligned}
$$

Thus, there exists a constant $L=L(\vartheta, \lambda) \geq 1$ such that

$$
(\operatorname{diam} f(B(x, \vartheta r)))^{2} \leq L \int_{B(x, r)} \mathcal{M}_{\Omega}^{2}(|D f|)(y) d y
$$

for each $x \in \Omega$ and all $r>0$ such that $B(x,(2+3 \vartheta) \sqrt{2} r) \subset \Omega$. Since $\mathcal{M}_{\Omega}^{2}(|D f|)$ is locally integrable by Lemma 3 , we have that

$$
\lim _{r \rightarrow 0} \int_{B(x, r)} \mathcal{M}_{\Omega}^{2}(|D f|)(y) d y=0 .
$$

Thus, for each $x \in \Omega$, there exists $R_{x}>0$ small enough to guarantee

$$
\int_{B(x, r)} \mathcal{M}_{\Omega}^{2}(|D f|)(y) d y<\frac{1}{L} \min \left\{1, e^{-\lambda}\right\},
$$

along with (10) for all $0<r<R_{x}$. This implies $\operatorname{diam}(f(B(x, \vartheta r))) \leq 1$ for all $0<r<R_{x}$. Using the monotonicity of the function $t \log ^{\lambda}(1 / t)$ 
for $t \in] 0, \min \left(1, e^{-\lambda}\right)$ [ together with $(10)$, we conclude

$$
\begin{aligned}
(\operatorname{diam} f & (B(x, \vartheta r)))^{2} \log ^{\lambda}\left(\frac{1}{\operatorname{diam} f(B(x, \vartheta r))}\right) \\
& \leq(\operatorname{diam} f(B(x, \vartheta r)))^{2} \log ^{\lambda}\left(\frac{1}{(\operatorname{diam} f(B(x, \vartheta r)))^{2}}\right) \\
& \leq L \log ^{\lambda}\left(\frac{1}{\int_{B(x, r)} \mathcal{M}_{\Omega}^{2}(|D f|)(z) d z}\right) \int_{B(x, r)} \mathcal{M}_{\Omega}^{2}(|D f|)(y) d y
\end{aligned}
$$

for all $r<R_{x}$ and the proposition follows.

Now we are ready to prove Theorem 2 .

Proof of Theorem 2. By the $\sigma$-additivity of the Hausdorff measure, we may assume that $E$ is contained in a square whose distance to the boundary of $\Omega$ is bounded away from zero. Let us assume in the following that all the appearing (also implicitly) balls and rings are contained in a cube $\Omega$ and $|D f| \log ^{\lambda}(e+|D f|)$ is integrable on $\Omega$. Applying Proposition 1 for $\vartheta=5$, we find a corresponding $R_{x}>0$ for each $x \in \Omega$. Setting $E_{n}:=\left\{x \in E: R_{x}<1 / n\right\}$, we see that $E=\cup E_{n}$. Thus, by the $\sigma$-additivity of the Hausdorff measure, it suffices to verify the theorem for bounded sets $E$ for which there exists a constant $R>0$ such that (9) holds with $\vartheta=5$ for each $x \in E$ and $r<R$.

Notice first that there exists $0<\alpha<2$ so that $\mathcal{H}^{g}(E)=0$, when $g(t)=t^{\alpha} \log ^{\lambda}\left(1 / t^{\alpha}\right)$. Indeed, let $\alpha=\operatorname{dim}_{\mathcal{H}}(E)+\varepsilon$, where $\varepsilon>0$ is chosen so that $\alpha<2$. Note that $\log ^{\lambda}(1 / t) \leq(1 / t)^{\varepsilon / 2}$ for $t$ small enough. We obtain

$$
t^{\alpha} \log ^{\lambda}\left(1 / t^{\alpha}\right) \leq \alpha^{\lambda} t^{\alpha} \log ^{\lambda}(1 / t) \leq \alpha^{\lambda} t^{\operatorname{dim}_{\mathcal{H}}(E)+\varepsilon / 2}
$$

for $t$ small enough. This proves the claim.

Let us fix $\delta_{1}>0$ and $\left.\varepsilon \in\right] 0, \min \left\{1, e^{-\lambda}\right\}\left[\right.$. We choose $\left.\delta_{0} \in\right] 0,5 R[$ such that

$$
f(B(x, 5 \rho)) \subset B\left(f(x), \delta_{1} / 2\right)
$$

for every $x \in E$ (the set $E$ is bounded and thus $f$ is uniformly continuous on a neighborhood of $E$ ) and all $0<\rho<\delta_{0} / 5<R$, where $R$ is the mentioned above constant for the set $E$. Since $E$ has zero $\mathcal{H}^{g}$-measure, we conclude by the Vitali Covering Theorem (see for example p. 27 in [EG92]) that there are countably many pairwise disjoint balls $B_{j}=B\left(x_{j}, r_{j}\right)$ such that

- $\sum r_{j}^{\alpha}<\sum r_{j}^{\alpha} \log ^{\lambda}\left(1 / r_{j}^{\alpha}\right)<\varepsilon$,

- $E \subset \cup B\left(x_{j}, 5 r_{j}\right)$ and

- $5 r_{j}<\delta_{0}$. 
We note that $f\left(B\left(x_{j}, 5 r_{j}\right)\right)$ is a $\delta_{1}$-cover of $f(E)$. Inequality (9) gives us the estimate

$$
\begin{aligned}
& \left(\operatorname{diam} f\left(B\left(x_{j}, 5 r_{j}\right)\right)\right)^{2} \log ^{\lambda}\left(\frac{1}{\operatorname{diam} f\left(B\left(x_{j}, 5 r_{j}\right)\right.}\right) \\
& \quad \leq L \log ^{\lambda}\left(\frac{1}{\int_{B\left(x_{j}, r_{j}\right)} \mathcal{M}_{\Omega}^{2}(|D f|)(y) d y}\right) \int_{B\left(x_{j}, r_{j}\right)} \mathcal{M}_{\Omega}^{2}(|D f|)(y) d y .
\end{aligned}
$$

Let us first consider the balls $B\left(x_{j}, r_{j}\right)$ that satisfy

$$
\int_{B\left(x_{j}, r_{j}\right)} \mathcal{M}_{\Omega}^{2}(|D f|)(y) d y \leq r_{j}^{\alpha} .
$$

As the function $t \log ^{\lambda}(1 / t)$ is increasing for $\left.t \in\right] 0, \min \left\{1, e^{-\lambda}\right\}[$, we conclude that

$$
\begin{aligned}
\log ^{\lambda}\left(\frac{1}{\int_{B\left(x_{j}, r_{j}\right)} \mathcal{M}_{\Omega}^{2}(|D f|)(z) d z}\right) \int_{B\left(x_{j}, r_{j}\right)} \mathcal{M}_{\Omega}^{2}(|D f|)(y) d y & \\
& \leq r_{j}^{\alpha} \log ^{\lambda}\left(\frac{1}{r_{j}^{\alpha}}\right) .
\end{aligned}
$$

If, on the other hand, $B\left(x_{j}, r_{j}\right)$ satisfies

$$
\int_{B\left(x_{j}, r_{j}\right)} \mathcal{M}_{\Omega}^{2}(|D f|)(y) d y>r_{j}^{\alpha},
$$

then

$$
\begin{aligned}
\int_{B\left(x_{j}, r_{j}\right)} \mathcal{M}_{\Omega}^{2}(|D f|)(y) & \log ^{\lambda}\left(\frac{1}{\int_{B\left(x_{j}, r_{j}\right)} \mathcal{M}_{\Omega}^{2}(|D f|)(z) d z}\right) d y \\
& \leq \int_{B\left(x_{j}, r_{j}\right)} \mathcal{M}_{\Omega}^{2}(|D f|)(y) \log ^{\lambda}\left(\frac{1}{r_{j}^{\alpha}}\right) d y .
\end{aligned}
$$

We split $B\left(x_{j}, r_{j}\right)$ into two parts $B_{1}$ and $B_{2}$, where

$$
\begin{aligned}
& B_{1}:=\left\{y \in B\left(x_{j}, r_{j}\right): \mathcal{M}_{\Omega}^{2}(|D f|)(y)<\frac{1}{r_{j}^{2-\alpha}}\right\}, \\
& B_{2}:=\left\{y \in B\left(x_{j}, r_{j}\right): \mathcal{M}_{\Omega}^{2}(|D f|)(y) \geq \frac{1}{r_{j}^{2-\alpha}}\right\} .
\end{aligned}
$$

We obtain the following two estimates $\left(r_{j}^{\alpha}<1\right)$ :

$$
\begin{aligned}
\int_{B_{1}} \mathcal{M}_{\Omega}^{2}(|D f|)(y) \log ^{\lambda}\left(\frac{1}{r_{j}^{\alpha}}\right) d y & \leq \int_{B_{1}} \frac{1}{r_{j}^{2-\alpha}} \log ^{\lambda}\left(\frac{1}{r_{j}^{\alpha}}\right) \\
& \leq C r_{j}^{\alpha} \log ^{\lambda}\left(\frac{1}{r_{j}^{\alpha}}\right)
\end{aligned}
$$


where $C$ is some constant independent of $x_{j}$ and $r_{j}$, and

$$
\begin{aligned}
\int_{B_{2}} \mathcal{M}_{\Omega}^{2} & (|D f|)(y) \log ^{\lambda}\left(\frac{1}{r_{j}^{\alpha}}\right) d y \\
& =\int_{B_{2}} \mathcal{M}_{\Omega}^{2}(|D f|)(y)\left(\frac{\alpha}{2-\alpha}\right)^{\lambda} \log ^{\lambda}\left(\frac{1}{r_{j}^{2-\alpha}}\right) d y \\
\leq & \int_{B_{2}} \mathcal{M}_{\Omega}^{2}(|D f|)(y)\left(\frac{\alpha}{2-\alpha}\right)^{\lambda} \log ^{\lambda}\left(\mathcal{M}_{\Omega}^{2}(|D f|)(y)\right) d y \\
\leq & \left(\frac{\alpha}{2-\alpha}\right)^{\lambda} \int_{B_{2}} \mathcal{M}_{\Omega}^{2}(|D f|)(y) \log ^{\lambda}\left(e+\mathcal{M}_{\Omega}^{2}(|D f|)(y)\right) d y .
\end{aligned}
$$

Let us set $r=\sup r_{j}$ and denote by $E_{r}$ the closed $r$-neighborhood of $E$. Lemma 3 gives us

$$
\int_{E_{r}} \mathcal{M}_{\Omega}^{2}(|D f|)(y) \log ^{\lambda}\left(e+\mathcal{M}_{\Omega}(|D f|)(y)\right) d y<\infty .
$$

Let $A_{r}:=\left\{x \in E_{r}: \mathcal{M}_{\Omega}(|D f|)(x) \geq \frac{1}{r^{1-\alpha / 2}}\right\}$. We obtain

$$
\int_{A_{r}} \mathcal{M}_{\Omega}^{2}(|D f|)(y) d y \geq \int_{A_{r}} \frac{1}{r^{2-\alpha}} d y=\mathcal{L}^{2}\left(A_{r}\right) \frac{1}{r^{2-\alpha}} .
$$

Consequently

$$
\mathcal{L}^{2}\left(A_{r}\right) \leq r^{2-\alpha} \int_{A_{r}} \mathcal{M}_{\Omega}^{2}(|D f|)(y) d y
$$

and thus $\lim _{r \rightarrow 0} \mathcal{L}^{2}\left(A_{r}\right)=0$. We obtain

$$
\begin{aligned}
\mathcal{H}_{\delta_{1}}^{h}(f(E)) & \leq \sum_{j=1}^{\infty}\left(\operatorname{diam} f\left(B\left(x_{j}, 5 r_{j}\right)\right)^{2} \log ^{\lambda}\left(\frac{1}{\operatorname{diam} f\left(B\left(x_{j}, 5 r_{j}\right)\right.}\right)\right. \\
& \leq C \int_{A_{r}}\left(\frac{\alpha}{2-\alpha}\right)^{\lambda} \mathcal{M}_{\Omega}^{2}(|D f|)(y) \log ^{\lambda}\left(e+\mathcal{M}_{\Omega}^{2}(|D f|)(y)\right) d y \\
& +C \sum_{j=1}^{\infty} r_{j}^{\alpha} \log ^{\lambda}\left(\frac{1}{r_{j}^{\alpha}}\right) .
\end{aligned}
$$

The integral above converges to zero as $\delta_{1}$ tends to zero. Then, assuming that,

$$
\int_{A_{r}}\left(\frac{\alpha}{2-\alpha}\right)^{\lambda} \mathcal{M}_{\Omega}^{2}(|D f|)(y) \log ^{\lambda}\left(e+\mathcal{M}_{\Omega}^{2}(|D f|)(y)\right) d y<\varepsilon,
$$

we obtain

$$
\mathcal{H}_{\delta_{1}}^{h}(f(E))<C \varepsilon .
$$

Letting first $\delta_{1}$ and then $\varepsilon$ go to zero, we get the claim.

Using the $\sigma$-additivity of the Hausdorff measure, we conclude with the following result. 
Corollary 1. Let $\Omega \subset \mathbb{R}^{2}$ be open and $f: \Omega \rightarrow f(\Omega)$ be a homeomorphism in $W^{1,2}\left(\Omega ; \mathbb{R}^{2}\right)$ and $|D f|^{2} \log ^{\lambda}(e+|D f|) \in L_{\text {loc }}^{1}\left(\Omega ; \mathbb{R}^{2}\right)$ for some $\lambda>0$. Assume that $E \subset \Omega$ is a countable union of sets of Hausdorff dimension strictly less than two. Then

$$
\mathcal{H}^{h}(f(E))=0
$$

for $h(t)=t^{2} \log ^{\lambda}\left(\frac{1}{t}\right)$.

\section{Proof of Theorem 1}

The combination of Theorem 2 with Theorem 1.1 in [AGRS] would give us Theorem 1 with $s<\lambda-1$ instead of $s<\lambda$. We employ a factorization trick to bridge this gap. The initial mapping $f$ will be decomposed into a quasiconformal mapping and a mapping with finite distortion, having better integrability properties than the distortion of the initial mapping. The tools used here are the Beltrami equation, Stoilow factorization (see, for example, [IM01], Chapter 11, or [Leh87], Chapter 4) and the so-called "minimal" decomposition for a Beltrami coefficient (see, for example, [Leh87, §4.7] and [Dav88, Proposition 3] for the quasiconformal case).

Consider the equation

$$
\bar{\partial} f(z)=\mu(z) \partial f(z)
$$

in the complex plane $\mathbb{C}$, where $\bar{\partial}=\frac{1}{2}\left(\partial_{x}+i \partial_{y}\right)$ and $\partial=\frac{1}{2}\left(\partial_{x}-i \partial_{y}\right)$. Equation (12) is called the Beltrami equation. The function $\mu$ is the Beltrami coefficient of the mapping $f$ (provided $f$ is a solution of (12) in some sense). Given an abstract Beltrami coefficient $\mu(z)$, such that $|\mu(z)|<1$ almost everywhere, we can associate to $\mu$ a real-valued function $K=\frac{1+|\mu|}{1-|\mu|}$, called a distortion function of the Beltrami coefficient. The terminology is natural, as the Beltrami equation yields the distortion inequality

$$
|D f(z)|^{2} \leq K(z) J_{f}(z)
$$

for its $W_{\text {loc }}^{1,1}$-solutions. Conversely, a mapping with finite distortion function $K(z)$ satisfies almost everywhere the Beltrami equation with the associated Beltrami coefficient $\mu_{f}(z)=\bar{\partial} f(z) / \partial f(z)$. In this case, $|\mu(z)| \leq \frac{K(z)-1}{K(z)+1}<1$ for almost every $z$.

The next lemma is essentially Corollary 4.4 from [AGRS].

Lemma 4. Let $\Omega \subset \mathbb{R}^{2}$ be a bounded domain and $f \in W_{\mathrm{loc}}^{1,1}\left(\Omega ; \mathbb{R}^{2}\right)$ be a mapping with $\lambda$-exponentially integrable distortion, $\lambda>0$. Given any $\mathbf{C}>1$, we can find a decomposition $f=h \circ g \circ f_{1}$ with a holomorphic $h: g\left(f_{1}(\Omega)\right) \rightarrow \mathbb{R}^{2}$, a $\mathbf{C}$-quasiconformal $g: f_{1}(\Omega) \rightarrow g\left(f_{1}(\Omega)\right)$ and $a$ homeomorphic $f_{1}: \Omega \rightarrow f_{1}(\Omega)$ of finite distortion, whose distortion is $\mathbf{C} \lambda$-exponentially integrable. 
Proof. We will think of $\Omega$ as of a domain in the complex plane $\mathbb{C}$ and consider $f$ as a complex mapping. Let $\mu$ and $K$ denote the Beltrami coefficient and the distortion function of $f$, respectively. Consider the Beltrami equation with the Beltrami coefficient $\mu=\mu_{f} \chi_{\Omega}$. By Theorem 11.8.3 in [IM01], this equation has a principal solution $f_{2}$ in the class $z+W_{\text {loc }}^{1, Q}(\mathbb{C}), Q(t)=\frac{t^{2}}{\log (e+t)}$, (i.e. $\left|\bar{\partial} f_{2}\right|+\left|\partial f_{2}-1\right| \in L^{Q}(\mathbb{C})$ ). See $§ 11.4$ in [IM01] for the definition of principal solution. In particular, $f_{2}$ is homeomorphic. Next, the mapping $f$ is a solution of the same equation a.e. in $\Omega$ and belongs to the Orlicz-Sobolev class $W_{\text {loc }}^{1, Q}(\mathbb{C})$ (see, for example, [IM01], §11.5). Thus, by Theorem 11.5.1 in [IM01], it can be represented as $f=h \circ f_{2}$, where $h: f_{2}(\Omega) \rightarrow \mathbb{C}$ is holomorphic. As a solution of the same Beltrami equation, $f_{2}$ satisfies

$$
\left|D f_{2}(z)\right|^{2} \leq K(z) J_{f_{2}}(z)
$$

almost everywhere in $\Omega$ and

$$
\left|D f_{2}(z)\right|^{2} \leq J_{f_{2}}(z)
$$

outside $\Omega$. By [AGRS, Corollary 4.4], $f_{2}$ can be represented as $f_{2}=$ $g \circ f_{1}$ in $\Omega$, where $g$ is $\mathbf{C}$-quasiconformal and $f_{1}$ is a homeomorphic mapping with finite, $\mathbf{C} \lambda$-exponentially integrable distortion. Thus,

$$
f=h \circ g \circ f_{1}
$$

gives us the desired decomposition.

In order to estimate the generalized Hausdorff measure of an image set under a quasiconformal mapping we employ the following lemma along with the higher regularity result for quasiconformal mappings from [Ast94].

Lemma 5. Let $g \in W_{\text {loc }}^{1, p}\left(\Omega^{\prime} ; \mathbb{R}^{2}\right)$ be continuous and $p>2$. If $F \subset \Omega^{\prime}$ satisfies $\mathcal{H}^{h}(F)=0$ for $h(t)=t^{2} \log ^{q}(1 / t), q>0$, then $\mathcal{H}^{\hat{h}}(g(F))=0$ for $\hat{h}=t^{2} \log ^{q(p-2) / p}(1 / t)$.

Proof. Let us fix a compact subset $\mathbf{K} \subset \subset \Omega^{\prime}$. It suffices to show that $\mathcal{H}^{\hat{h}}(g(F \cap \mathbf{K}))=0$. Pick an open set $G \subset \subset \Omega^{\prime}$, containing the set $F \cap \mathbf{K}$. As $\mathcal{H}^{h}(F \cap \mathbf{K})=0$, given any $\varepsilon>0$, we can choose a covering $\mathcal{Q}=$ $\left\{Q_{i} \subset G: i \in \mathbb{N}\right\}$ of $F \cap \mathbf{K}$ by closed squares, having pairwise disjoint interiors, whose diameters $l_{i}, i \in \mathbb{N}$, satisfy $l_{i}<\min \left\{1, e^{-\frac{(p-2 q)}{2 p}}\right\}$, and such that

$$
\sum_{i=1}^{\infty} l_{i}^{2} \log ^{q} \frac{1}{l_{i}}<\varepsilon
$$

We have $g(F \cap \mathbf{K})) \subset \cup_{i} g\left(Q_{i}\right)$. Morrey's inequality gives us (see, for example, [EG92, p. 143])

$$
\operatorname{diam} g\left(Q_{i}\right) \leq C_{p}\left(\operatorname{diam}\left(Q_{i}\right)\right)^{1-\frac{2}{p}}\left(\int_{Q_{i}}|D g(y)|^{p} d y\right)^{1 / p}
$$


for $i=1,2, \ldots$. Using the monotonicity of the function $\hat{h}(t)$ for $t \in$ ] $0, \min \left\{1, e^{-\frac{(p-2) q}{2 p}}\right\}[$, we estimate

$$
\begin{aligned}
\sum_{i=1}^{\infty} \hat{h} & \left(\operatorname{diam} g\left(Q_{i}\right)\right) \\
& \leq \sum_{\left\{i: \operatorname{diam} g\left(Q_{i}\right) \leq l_{i}\right\}} \hat{h}\left(\operatorname{diam} g\left(Q_{i}\right)\right)+\sum_{\left\{i: \operatorname{diam} g\left(Q_{i}\right)>l_{i}\right\}} \hat{h}\left(\operatorname{diam} g\left(Q_{i}\right)\right) \\
& \leq \sum_{i=1}^{\infty} \hat{h}\left(l_{i}\right)+\sum_{i=1}^{\infty} \operatorname{diam}^{2} g\left(Q_{i}\right) \log ^{q(p-2) / p}\left(\frac{1}{l_{i}}\right) \\
& \leq \sum_{i=1}^{\infty} h\left(l_{i}\right)+C_{p}^{2} \sum_{i=1}^{\infty} l_{i}^{\frac{2(p-2)}{p}}\left(\int_{Q_{i}}|D g(y)|^{p} d y\right)^{2 / p} \log ^{q(p-2) / p}\left(\frac{1}{l_{i}}\right) \\
& \leq \sum_{i=1}^{\infty} h\left(l_{i}\right)+\tilde{C}\left(\sum_{i=1}^{\infty} h\left(l_{i}\right)\right)^{\frac{p-2}{2}}\left(\sum_{i=1}^{\infty} \int_{Q_{i}}|D g(y)|^{p} d y\right)^{2 / p} \\
& \leq \varepsilon+\tilde{C} \varepsilon^{\frac{p-2}{p}}\left(\int_{G}|D g(y)|^{p} d y\right)^{2 / p},
\end{aligned}
$$

where the third step is due to Morrey's inequality and the fact that $\frac{q(p-2)}{p}<q$ and the second to last step is simply the Hölder inequality for series. Letting $\varepsilon \rightarrow 0$ completes the proof.

We are now ready to prove Theorem 1.

Proof of Theorem 1. It is enough to show that $\mathcal{H}^{h_{s}}\left(f\left(E \cap \Omega_{1}\right)\right)=0$ for each $s \in] 0, \lambda\left[\right.$ and each domain $\Omega_{1} \subset \subset \Omega$. Fix such an $s$ and $\Omega_{1}$. Let us take a factorization $f=h \circ g \circ f_{1}$ in $\Omega_{1}$ as in Lemma 4 for $\mathbf{C}>\max \{1,1 /(\lambda-s)\}$. By Theorem 1.1 from [AGRS], we have $\left|D f_{1}\right|^{2} \log ^{q}\left(e+\left|D f_{1}\right|\right) \in L_{\text {loc }}^{1}\left(\Omega_{1}\right)$ for all $q<\mathbf{C} \lambda-1$, as the distortion of $f_{1}$ is $\mathbf{C} \lambda$-exponentially integrable in $\Omega_{1}$. Theorem 2 implies $\mathcal{H}^{h_{q}}\left(f_{1}\left(E \cap \Omega_{1}\right)\right)=0$ for each $\left.q \in\right] 0, \mathbf{C} \lambda-1[$. In order to combine this with Lemma 5 , we note that $g \in W_{\text {loc }}^{1, p}\left(f_{1}\left(\Omega_{1}\right)\right)$ for all $p<2 \mathbf{C} /(\mathbf{C}-1)$ [Ast94, Corollary 1.2], as $g$ is $\mathbf{C}$-quasiconformal in $f_{1}\left(\Omega_{1}\right)$. Thus, by Lemma 5, we have

$$
\mathcal{H}^{h_{s_{1}}}\left(g\left(f_{1}\left(E \cap \Omega_{1}\right)\right)\right)=0
$$

where $s_{1}=\frac{(p-2) q}{p}$, for all $p<2 \mathbf{C} /(\mathbf{C}-1)$ and $\left.q \in\right] 0, \mathbf{C} \lambda-1[$. In other words, (13) holds for all $s_{1}>0$ such that

$$
s_{1}<(\mathbf{C} \lambda-1)\left(\frac{2 \mathbf{C}}{\mathbf{C}-1}-2\right) \frac{\mathbf{C}-1}{2 \mathbf{C}}=\lambda-\frac{1}{\mathbf{C}},
$$

and thus, for $s_{1}=s$. Finally, as $h$ is holomorphic in $\Omega_{1}$, and thus, locally Lipschitz, we obtain $\mathcal{H}^{h_{s}}\left(f\left(E \cap \Omega_{1}\right)\right)=0$. 


\section{REFERENCES}

[AGRS] K. Astala, J. Gill, S. Rohde, and E. Saksman. Optimal regularity for planar mappings of finite distortion. Ann. Inst. H. Poincaré Anal. Non Linéaire. to appear.

[Ast94] K. Astala. Area distortion of quasiconformal mappings. Acta Math., 173(1):37-60, 1994.

[Boy57] B. V. Boyarskiǔ. Generalized solutions of a system of differential equations of first order and of elliptic type with discontinuous coefficients. Mat. Sb. N.S., 43(85):451-503, 1957.

[Dav88] G. David. Solutions de l'équation de Beltrami avec $\|\mu\|_{\infty}=1$. Ann. Acad. Sci. Fenn. Ser. A I Math., 13(1):25-70, 1988.

[EG92] L. C. Evans and R. F. Gariepy. Measure theory and fine properties of functions. Studies in Advanced Mathematics. CRC Press, Boca Raton, FL, 1992.

[FKZ05] D. Faraco, P. Koskela, and X. Zhong. Mappings of finite distortion: the degree of regularity. Adv. Math., 190(2):300-318, 2005.

[GIM95] L. Greco, T. Iwaniec, and G. Moscariello. Limits of the improved integrability of the volume forms. Indiana Univ. Math. J., 44(2):305-339, 1995.

[GV73] F. W. Gehring and J. Väisälä. Hausdorff dimension and quasiconformal mappings. J. London Math. Soc. (2), 6:504-512, 1973.

[HK03] D. A. Herron and P. Koskela. Mappings of finite distortion: gauge dimension of generalized quasicircles. Illinois J. Math., 47(4):1243-1259, 2003.

[IKM02] T. Iwaniec, P. Koskela, and G. Martin. Mappings of BMO-distortion and Beltrami-type operators. J. Anal. Math., 88:337-381, 2002. Dedicated to the memory of Tom Wolff.

[IKMS03] T. Iwaniec, P. Koskela, G. Martin, and C. Sbordone. Mappings of finite distortion: $L^{n} \log ^{\chi} L$-integrability. J. London Math. Soc. (2), 67(1):123136, 2003.

[IKO01] T. Iwaniec, P. Koskela, and J. Onninen. Mappings of finite distortion: monotonicity and continuity. Invent. Math., 144(3):507-531, 2001.

[IM01] T. Iwaniec and G. Martin. Geometric function theory and non-linear analysis. Oxford Mathematical Monographs. The Clarendon Press Oxford University Press, New York, 2001.

[IM08] T. Iwaniec and G. Martin. The Beltrami equation. Mem. Amer. Math. Soc., 191(893):x+92, 2008.

[Kau00] R. Kaufman. Sobolev spaces, dimension, and random series. Proc. Amer. Math. Soc., 128(2):427-431, 2000.

[KKM01a] J. Kauhanen, P. Koskela, and J. Malý. Mappings of finite distortion: condition N. Michigan Math. J., 49(1):169-181, 2001.

[KKM01b] J. Kauhanen, P. Koskela, and J. Malý. Mappings of finite distortion: discreteness and openness. Arch. Ration. Mech. Anal., 160(2):135-151, 2001.

[KZZ $\quad$ P. Koskela, A. Zapadinskaya, and T. Zürcher. Generalized dimension distortion under planar Sobolev homeomorphisms. in preparation.

[Leh87] O. Lehto. Univalent functions and Teichmüller spaces, volume 109 of Graduate Texts in Mathematics. Springer-Verlag, New York, 1987.

[Reš66] Ju. G. Rešetnjak. Certain geometric properties of functions and mappings with generalized derivatives. Sibirsk. Mat. Z̆., 7:886-919, 1966. 
(Pekka Koskela) Department of Mathematics and Statistics, UniverSITY OF JyvÄSkylä, P.O. BoX 35, Fin-40014 University of Jyvëskylä, FINLAND

E-mail address: pkoskela@maths.jyu.fi

(Aleksandra Zapadinskaya) Department of Mathematics and Statistics, UNiversity of JyvÄSKYlä, P.O. Box 35, Fin-40014 UnivERSity OF JyVÄSKYLÄ, FINLAND

E-mail address: alzapadi@cc.jyu.fi

(Thomas Zürcher) Mathematical Institute, University of Bern, SidlerSTRASSE 5, CH-3012 Bern, Switzerland

E-mail address: thomas.zuercher@math.unibe.ch 